\title{
КАЛИБРОВКА ОПТИЧЕСКИХ СИСТЕМ ДЛЯ ИЗМЕРЕНИЯ РАСХОДИМОСТИ ЛАЗЕРНОГО ИЗЛУЧЕНИЯ
}

\author{
(Представил Х. Абен)
}

Важным параметром лазерного излучения является его расходимость. Лазерное излучение с малой угловой расходимостью необходимо при использовании лазеров в различных интерферометрических приложениях в голографии, для дистанционного исследования объектов, в лазерной технологии и др.

Под расходимостью лазерного излучения $\theta$ понимается плоский или телесный угол, «характеризующий ширину диаграммы направленности лазерного излучения в дальней зоне по заданному уровню углового распределения энергии или мощности лазерного излучения, определяемому по отношению к его максимальному значению» [1 ${ }^{1}$. Выходное излучение лазеров с устойчивыми резонаторами, работающих в одномодовом режиме, представляет собой гауссов пучок $\left[{ }^{2}\right]$. В этом случае условие дальней зоны имеет следующий вид $\left[{ }^{3}\right]$ :

$$
L \gg z_{0}
$$

где $z_{0}-$ конфокальный параметр пучка, $L-$ расстояние от перетяжки пучка до плоскости измерений.

Асимптотический угол расходимости гауссова пучка $\theta_{\mathrm{pacx}}$ (по уровню $1 / e^{2}$ ) определяется по следующей формуле:

$$
\theta_{\mathrm{pacx}}= \pm \sqrt{\frac{\lambda}{\pi z_{0}}}
$$

где $\lambda$ - длина волны излучения лазера.

При измерениях расходимости лазерного излучения основное применение нашли метод сечений и метод фокального пятна $\left[{ }^{4}\right]$. Однако если конфокальный параметр пучка $z_{0}$ имеет величину порядка нескольких метров, измерения методом сечений следует производить на значительных расстояниях от излучателя. В этом случае единственно приемлемым является метод фокального пятна, согласно которому угол расходимости излучения лазера $\theta$ измеряется в фокальной плоскости объекта с фокусным расстоянием $f$ по формуле

$$
\theta=\operatorname{arctg} d_{0} / f,
$$

где $d_{0}$ - диаметр сечения пучка в фокальной плоскости объектива (диаметр фокального пятна).

Из последней формулы следует, что для измерения узконаправленного лазерного излучения требуется длиннофокусная оптика. В противном случае возникают проблемы с подбором высокоразрешающих регистрирующих сред. 
Известно, что одним из факторов, ограничивающих точность измерения расходимости излучения методом фокального пятна, является ошибка определения положения фокальной плоскости измерительной системы $\left[{ }^{5}\right]$. Другой источник погрешностей - неточность определения величины фокусного расстояния оптической системы, используемой при измерениях. Для учета указанных погрешностей по интерференционным

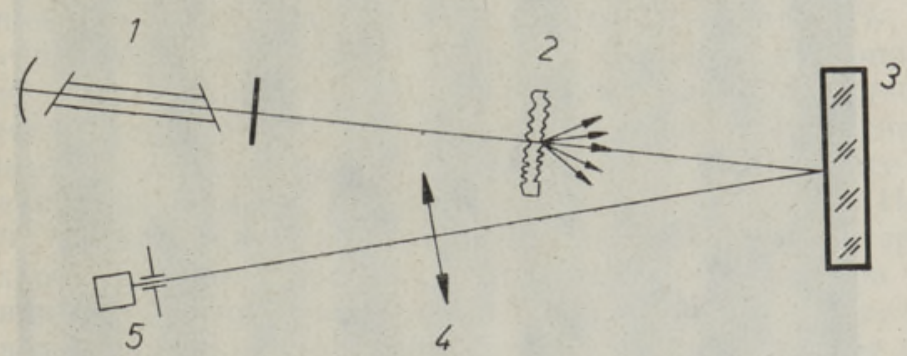

Рис. 1. Схема калибровки оптических систем, 1 - лазер, 2 - диффузный рассеиватель, 3 - плоско-параллельная пластина, 4 - длиннофокусная линза, 5 - фотодиод с точечной диафрагмой.

полосам равного наклона производилась калибровка длиннофокусной оптической системы, служившей для измерения расходимости лазерного излучения методом фокального пятна (рис. 1). Излучение лазера 1 направлялось на диффузный рассеиватель 2 (матовое стекло) и далее попадало на плоско-параллельную пластину 3 . Отраженное пластиной 3 излучение проходило через оптическую систему 4 , положение фокальной плоскости и фокусное расстояние которой требовалось определить. В фокальной нлоскости оптической системы наблюдались интерференционные полосы равного наклона, которые регистрировались фотодиодом с гочечной диафрагмой 5 , а также фотографировались (см. рис. 2).

Условие максимумов при получении интерференционных полос равного наклона имеет следующий вид $\left[{ }^{6}\right]$

$$
2 t \sqrt{n^{2}-\sin ^{2} \alpha=} \pm\left(m-\frac{1}{2}\right) \lambda
$$

где $t$ - толщина плоско-параллельной пластины; $n$ - показатель преломления материала пластины; $\alpha$ - угол падения (отражения); $m-$ порядок интерференции.

Из формулы (4) легко получить, что расстояние $\delta$ между максимумами полос равного наклона в фокальной плоскости исследуемой линзы следующее:

$$
\delta=\lambda f \frac{\sqrt{n^{2}-\sin ^{2} \alpha}}{t \sin 2 \alpha} .
$$

В качестве длиннофокусной оптической системы использовалась линза с фокусным расстоянием около 4 м. Положение фокальной плоскости определялось по максимальному контрасту интерференционных полос равного наклона (см. рис. $2, a)$. При смешении из положения фокальной плоскости контраст полос вначале уменьшался (рис. 2,б), а затем полосы исчезали. Величина фокусного расстояния линзы определялась двумя способами.

1. Непосредственным измерением отрезка вдоль оптической оси от фокальной плоскости до задней поверхности линзы. Правомерность такого измерения вытекает из того, что при малых кривизнах оптических по- 
верхностей рабочий отрезок практически совпадает с фокусным расстоянием, т. е. линза является «тонкой». Величина фокусного расстояния, полученная в результате измерений, равна $3,99 \pm 0,04$ м.
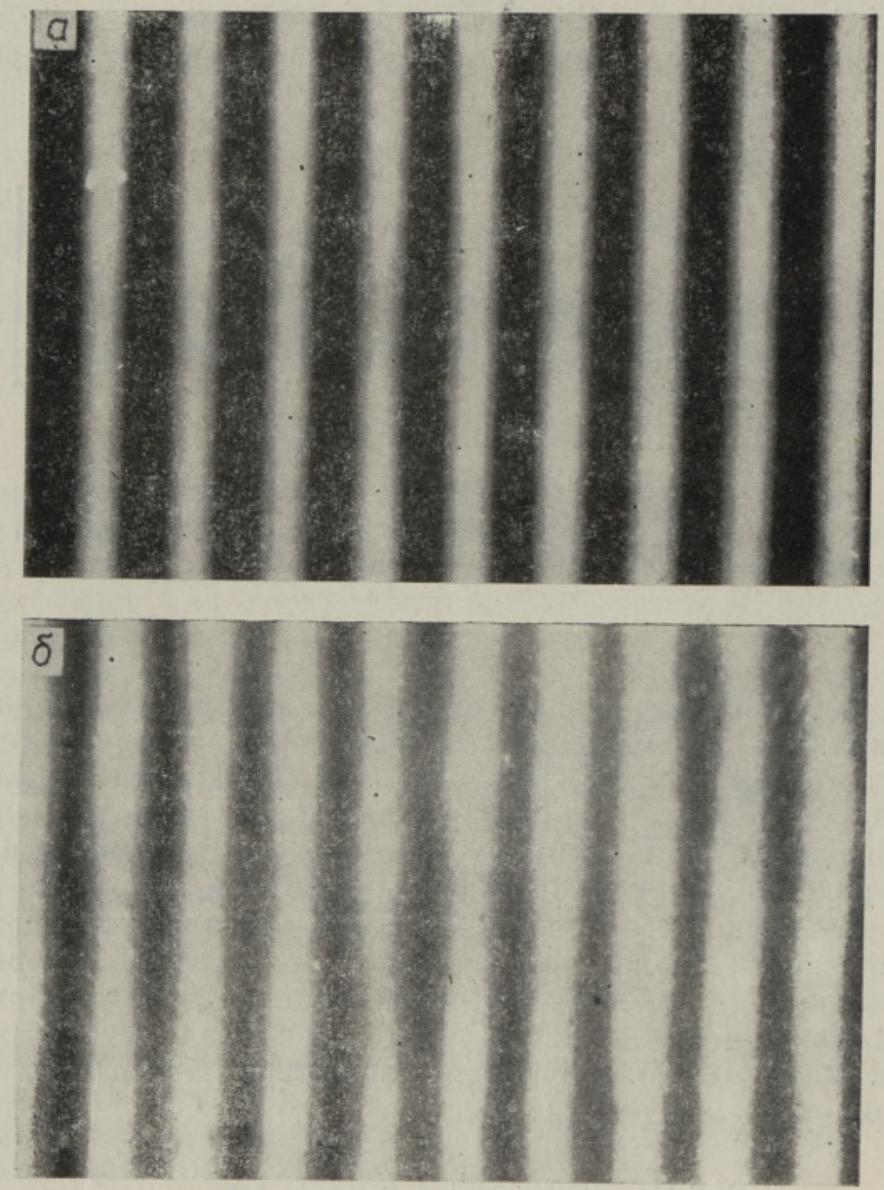

Рис. 2. Полосы равного наклона: $a-$ в фокальной плоскости линзы, $б-$ смещенные из положения фокальной плоскости.

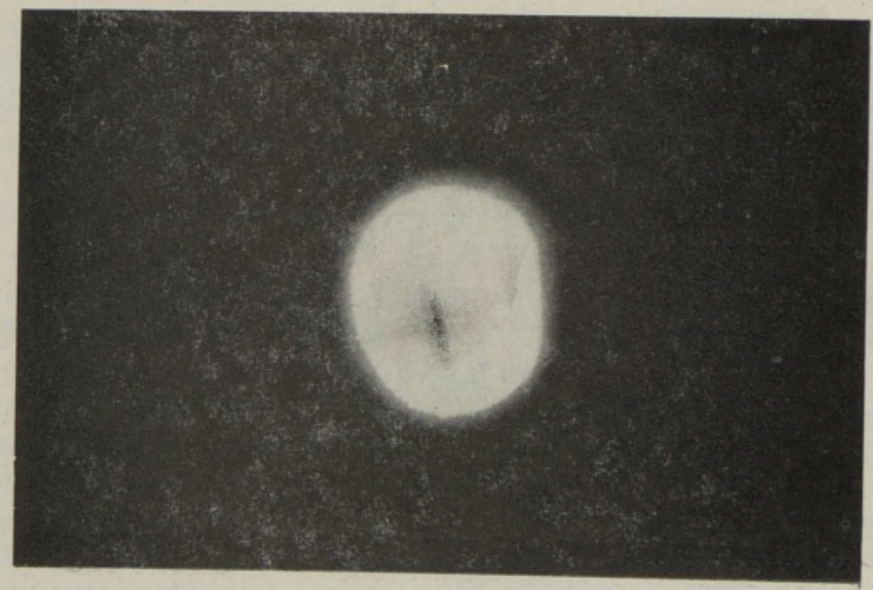

Рис. 3. Фокальное пятно, 
2. Путем расчета по формуле, следующей из соотношения (5)

$$
f=\frac{\delta t \sin 2 \alpha}{\lambda \sqrt{n^{2}-\sin ^{2} \alpha}} .
$$

С учетом погрешностей измерения величин, входящих в формулу (6), расчётное значение фокусного расстояния получилось равным $4,08+-$ \pm 0.11 м, при этом относительная погрешность составила $2,7 \%$. Основной вклад в указанную погрешность $(\simeq 1,4 \%)$ обусловлен неопределенностью в измерении угла $\alpha$. Отметим, что относительная погрешность определения фокусных расстояний длиннофокусных оптитеских систем с помощью специального оборудования $\left[{ }^{7}\right]$, как правнло, не менее $1 \%$.

Измерение расходимости производилось на одномодовом $\mathrm{He}-\mathrm{Ne}$-лазере ЛГ-38, обладающем наилучшей направленностыю из серийно выпускаемых отечественной промышленностью $\mathrm{He}-\mathrm{Ne}$-лазеров (паспортное значение расходимости не более 0,5 мрад). Фокальное пятно, полученное с помощью длиннофокусной линзы и представленное на рис. 3 имеет форму эллипса с размерами по главным осям $2,24 \pm 0,03$ и $1,97 \pm 0,03$ мм, измеренным по уровню $1 / e^{2}$. Этому соответствует расходимость излучения соответственно 0,56 и 0,49 мрад. Такая деформация основной моды обусловлена, видимо, разъюстировкой лазерного резонатора.

Относительная погрешность измерения расходимости излучения методом фокального пятна с учетом погрешностей измерения размеров фокального пятна, величины фокусного расстояния линзы. а также определения положения фокальной плоскости в нашем случае составила $5,2 \%$.

Выводы. Калибровка длиннофокусных оптических систем по интерференционным полосам равного наклона не требует специального оборудования и может быть реализована практически в любой лабораторин. Достигнутая при этом точность определения фокусного расстояния и положения фокальной плоскости обеспечила измерение расходимости узконаправленного лазерного излучения с относительной погрешностью $5 \%$. Использование более длиннофокусной оптики позволит проводить измерение расходимости лазерного излучения с еще более высокой точностью.

\section{Л ИТ Е РА Т У Р А}

1. ГОСТ 24453-80. Измерения параметроз и характеристик лазерного излучения. Термины, определения и буквенные обозначения.

2. Kogelnik, $H_{\text {. }} L i, T$. Appl. Opt., 5, № 10, 1550-1567 (1966).

3. Джеррард А., Берч Дж. М. Введение в матричную оптику. М., «Мир», 1978.

4. Измерение энергетических параметроз и характеристик лазерного и лучения. Пол ред. А. Ф. Котюка. М. «Радио н гзязь», 1981.

5. Webb, W. E. Appl. Opt., 17, № 5, 685-686 (1978).

6. Борн М., Вольф Э. Основы оптикн. М., «Наука», 1973.

7. Афанасьев В. А. Оптические измерения. М., «Высшая школаз, 1981.

Ннститут кибернетики

Академии наук Эстонской ССР

Специальное конструкторское бюро

Академии наук Эстонской ССР

\section{Поступила в редлкцию} 15/XI 1985 


\section{LASERKIIRE LAHKNEVUSE MOOOTMISE OPTILISE SOSTEEMI KALIBREERIMINE}

On esitatud ühemoodilise He-Ne-laseri ЛГ-38 kiire lahknevuse mõõtmise tulemused ja kirjeldatud meetodit, mida kasutatakse pikafookuselise süsteemi kalibreerimisel. Meetodi kõige olulisem eelis on, et ta vōimaldab kalibreerimist ilma keeruliste seadmeteta, Rakendades 4-meetrilist optilist süsteemi sai kiire lahknevust mõōta 5,2\% täpsusega,

\section{K.-J. KELL, J. SOSKIND \\ OPTICAL SYSTEM CALIBRATION FOR LASER BEAM DIVERGENCE MEASUREMENT}

The results of beam divergence measurements of a single mode He-Ne laser ЛГ-38, are presented. The practical problems with the use of a long-focus optical system in beam divergence measurements are to locate the focal plane and to measure the focal length. The location of the focal plane is found using the interference fringes generated in the focal plane of the long-focal system by radiation reflected from an uncoated etalon. The system is finely focused by adjusting the separation to the receiver for maximum fringe sharpness. Each fringe is emitted from the etalon at a known angle, according to formula (5). Thus the focal length is calculated by formula (6), which follows directly from (5). The main advantage of the long-focus system calibration described in this paper lies in the fact that no sophisticated equipment is needed. By means of a 4 meter optical system the laser beam divergence was measured with $5.2 \%$ accuracy. 